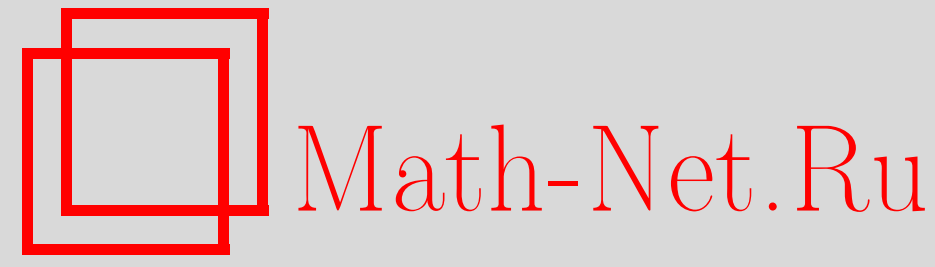

S. Luo, On Covariance and Quantum Fisher Information, Теория вероятн. и ее примен., 2008, том 53, выпуск $2,393-397$

DOI: https://doi.org/10.4213/tvp2423

Использование Общероссийского математического портала Math-Net.Ru подразумевает, что вы прочитали и согласны с пользовательским соглашением

http://www . mathnet.ru/rus/agreement

Параметры загрузки:

IP : 18.208 .226 .222

26 апреля 2023 г., 08:37:58

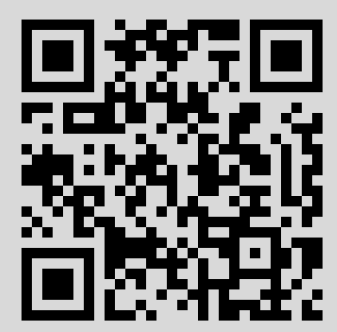


3. Ротарь В.И. Неклассические оценки скорости сходимости в многомерной центральной предельной теореме. I, II. - Теория вероятн. и ее примен., 1977, т. 22, в. 4 , c. $774-790 ; 1978$, т. 23 , в. 1 , c. $55-66$.

4. Sazonov V.V., Ul'yanov V.V. On the accuracy of normal approximation. - J. Multivariate Anal., 1982, v. 12, № 3, p. 371-384.

5. Бенткус $B$. Новый подход к аппроксимациям в теории вероятностей и теории операторов. - Liet. Matem. Rink., 2003, т. 43, № 4, c. 444-470.

6. Петров $B$. В. Предельные теоремы для сумм независимых случайных величин. М.: Наука, 1987, 317 с.

Поступила в редакцию 16.V.2008

(C) $2008 \mathrm{r}$.

LUO S.* $^{*}$

\section{ON COVARIANCE AND QUANTUM FISHER INFORMATION ${ }^{1)}$}

Для квантового состояния и множества наблюдаемых можно построить ассоциированную матрицу ковариаций и естественную матрицу квантовой информации Фишера. Эти две матрицы характеризуют неопределенность и информацию, содержашиеся в наблюдаемых для соответствуюшего состояния. В статье установлено неравенство между двумя матрицами. Это неравенство можно интерпретировать как обшее квантование принципа неопеделенности Гейзенберга с точки зрения статистического оценивания. В частности, из этого неравенства следует новое соотношение неопределенности, которое уточняет знаменитое соотношение неопределенности Шрёдингера.

Ключевые слова и фразы: ковариация, квантовая информация Фишера, соотношения неопределенности, детерминант.

1. Introduction. The mathematical aspect of quantum mechanics, conveniently formulated in a Hilbert space framework [15], can be most efficiently and elegantly interpreted in the language of probability and statistics [9]. In generic situations, there are irreducible randomness in both quantum states, which are represented by density operators (positive operators with unit trace), and observables, which are represented by self-adjoint operators. For simplicity, we will work in finite dimensional Hilbert spaces. However, in principle we can follow the method given in [9] to generalize these arguments to embrace infinite dimensional cases.

Given a quantum state $\rho$ and a set of observables $H_{1}, \ldots, H_{n}$, the covariance matrix $\mathbf{C}:=\left(C_{\alpha \beta}\right)_{1 \leqslant \alpha, \beta \leqslant n}$ is defined as

$$
C_{\alpha \beta}:=\operatorname{cov}_{\rho}\left(H_{\alpha}, H_{\beta}\right) \equiv \operatorname{tr} \rho H_{\alpha} H_{\beta}-\left(\operatorname{tr} \rho H_{\alpha}\right)\left(\operatorname{tr} \rho H_{\beta}\right), \quad \alpha, \beta=1, \ldots, n .
$$

Here $\operatorname{tr}$ denotes trace. In particular, $V\left(\rho, H_{\alpha}\right):=C_{\alpha \alpha}$ is the variance of the observable $H_{\alpha}$ in the state $\rho$. It should be remarked that the matrix (1) is only a formal analog of the classical covariance matrix, and it does not refer to a single quantum measurement.

In the context of two observables which are often incompatible (non-commutative), the celebrated Heisenberg uncertainty relation (cf. [1], [9])

$$
V\left(\rho, H_{1}\right) V\left(\rho, H_{2}\right) \geqslant \frac{1}{4}\left|\operatorname{tr} \rho\left[H_{1}, H_{2}\right]\right|^{2}
$$

is often interpreted as setting a fundamental limit to the accuracy of simultaneous measurement of the observables $H_{1}$ and $H_{2}$ in terms of their commutator $\left[H_{1}, H_{2}\right]:=H_{1} H_{2}-H_{2} H_{1}$,

* Academy of Mathematics and Systems Science, Chinese Academy of Sciences, 100080 Beijing, P. R. China; e-mail: luosl@amt.ac.cn

1) This work is supported by NSFC (grants № 10571166 and 10771208) and by Science Fund for Creative Research Groups (grant № 10721101). 
which is a basic character in quantum mechanics. However, this interpretation is somewhat inconsistent as incisively analyzed in [9, p. 70-73], a more rigorous interpretation of relation (2) is that it characterizes variances of two different incompatible measurements (of $H_{1}$ and $H_{2}$ ) related to the same quantum state $\rho$, rather than joint approximate measurement of $H_{1}$ and $H_{2}$ [9].

Later, Schrödinger refined the uncertainty relation (2) to

$$
V\left(\rho, H_{1}\right) V\left(\rho, H_{2}\right) \geqslant \frac{1}{4}\left(\left|\operatorname{tr} \rho\left[H_{1}, H_{2}\right]\right|^{2}+\left|\operatorname{tr} \rho\left\{H_{1}^{\prime}, H_{2}^{\prime}\right\}\right|^{2}\right)
$$

(cf. [13], [14]). Here $\left\{H_{1}^{\prime}, H_{2}^{\prime}\right\}:=H_{1}^{\prime} H_{2}^{\prime}+H_{2}^{\prime} H_{1}^{\prime}$ denotes anticommutator, and $H_{1}^{\prime}:=$ $H_{1}-\left(\operatorname{tr} \rho H_{1}\right) 1, H_{2}^{\prime}:=H_{2}-\left(\operatorname{tr} \rho H_{2}\right) 1$, and 1 is the identity operator. It is remarkable that the anticommutator is intimately related to the real part of the covariance $\operatorname{Cov}_{\rho}(\cdot, \cdot)$ :

$$
\operatorname{tr} \rho\left\{H_{1}^{\prime}, H_{2}^{\prime}\right\}=\operatorname{Cov}_{\rho}\left(H_{1}, H_{2}\right)+\operatorname{cov}_{\rho}\left(H_{2}, H_{1}\right)=2 \operatorname{Re}_{\operatorname{cov}}\left(H_{1}, H_{2}\right)
$$

and the commutator is intimately related to the imaginary part of the covariance:

$$
\operatorname{tr} \rho\left[H_{1}, H_{2}\right]=\operatorname{cov}_{\rho}\left(H_{1}, H_{2}\right)-\operatorname{cov}_{\rho}\left(H_{1}, H_{2}\right)=2 i \operatorname{Im} \operatorname{cov}_{\rho}\left(H_{1}, H_{2}\right) .
$$

Here Re and Im denote the real part and imaginary part of a complex number, respectively. Accordingly, the Schrödinger uncertainty relation (3) is apparently stronger than the Heisenberg uncertainty relation (2), and it sets a limit to quantum measurement by incorporating both the incompatibility (as quantified by the commutator) and the correlation (as quantified by the anticommutator) between the observables $H_{1}$ and $H_{2}$.

Now taking into account (4) and (5), the Schrödinger uncertainty relation (3) can be rewritten as

$$
V\left(\rho, H_{1}\right) V\left(\rho, H_{2}\right)-\left|\operatorname{cov}_{\rho}\left(H_{1}, H_{2}\right)\right|^{2} \geqslant 0 .
$$

The covariance matrix as defined by (1) is clearly positive, this seemingly simple fact already implies the uncertainty relations (2) and (6). From the general spirit of the Heisenberg uncertainty principle, it is reasonable to expect that the covariance matrix can be bounded by some positive matrix from below. This paper is devoted to establishing such a lower bound from an informational approach. We will prove a matrix inequality between the covariance matrix and the quantum Fisher information matrix involving the symmetric logarithmic derivative. As a simple consequence by taking determinant of positive matrices, we readily obtain an uncertainty relation which is stronger than inequality (6), and moreover possesses an intuitive informational interpretation.

2. Quantum Fisher information. First we recall some notation and notions. We will use the standard Dirac notion of ket $|\psi\rangle$ for representing a vector in a finite dimensional Hilbert space $\mathscr{H}[6],[9],[13]$, the adjoint of $|\psi\rangle$ is called a bra and denoted by $\langle\psi|$. The inner product in $\mathscr{H}$ is denoted by $\langle\psi \mid \phi\rangle$, which is equivalent to the usual mathematical notation $\langle\psi, \phi\rangle$. Moreover, for an operator $A$ on $\mathscr{H},\langle\psi|A| \phi\rangle$ just means mathematically $\langle\psi, A \phi\rangle$. By $|\psi\rangle\langle\psi|$ we mean the rank one operator projecting on $|\psi\rangle$. The advantage of the notation lies in the convenience in manipulating the products of bra, ket, and operators.

A matrix $A$ is called positive if $\langle\psi|A| \psi\rangle \geqslant 0$ for any vector $|\psi\rangle$. The matrix inequality $A \geqslant B$ means that $A-B$ is positive.

In order to improve inequality (6) and to understand the Heisenberg uncertainty principle from a more general perspective, we will take an informational approach. The idea is to use a quantum analogue of the classical Fisher information matrix to set a positive lower bound to the covariance matrix $\mathbf{C}$. This will in particular imply a lower bound to the quantity $V\left(\rho, H_{1}\right) V\left(\rho, H_{2}\right)-\left|\operatorname{cov}_{\rho}\left(H_{1}, H_{2}\right)\right|^{2}$ in the left-hand side of inequality (6).

To motivate our consideration, let us recall the definition of the classical Fisher information matrix. For a family of probability densities $\left\{p_{\theta}\right\}$ on $\mathbf{R}^{m}$ with parameters $\theta=\left(\theta_{1}, \theta_{2}, \ldots, \theta_{n}\right) \in \mathbf{R}^{n}$ satisfying some regularity conditions, its Fisher information matrix $\mathbf{J}_{\theta}:=\left(J_{\alpha \beta}\right)_{1 \leqslant \alpha, \beta \leqslant n}$ is defined by

$$
J_{\alpha \beta}:=\int \frac{\partial \ln p_{\theta}(x)}{\partial \theta_{\alpha}} \frac{\partial \ln p_{\theta}(x)}{\partial \theta_{\beta}} p_{\theta}(x) d x
$$

(see [5], [7]). Of course, the above quantity usually depends on the set of parameters $\theta$. But in the particular and important case when $m=n$ and $\theta$ is a set of shift parameters in 
the sense that $p_{\theta}(x)=p(x-\theta)$ for some probability density $p(x)$, the Fisher information matrix $\mathbf{J}_{\theta}$ is independent of the parameters $\theta$ and, due to the translation invariance of the Lebesgue integral, it can be expressed as

$$
J_{\alpha \beta}=\int \frac{\partial \ln p(x)}{\partial x_{\alpha}} \frac{\partial \ln p(x)}{\partial x_{\beta}} p(x) d x
$$

The quantum analogues of a probability density $p$ and the integration are a density operator $\rho$ and the trace, respectively. In noncommutative probability and quantum calculus, the derivative of a state $\rho$ with respect to an observable $H_{\alpha}$ is represented by the commutator [4]

$$
D_{H_{\alpha}} \rho:=i\left[\rho, H_{\alpha}\right] .
$$

This is the quantum analogue of the classical derivative $\partial p(x) / \partial x_{\alpha}$.

The classical logarithmic derivative $\partial \ln p(x) / \partial x_{\alpha}$ has many different quantum analogues. One distinguished variant with special significance in the quantum detection and estimation theory is the so-called symmetric logarithmic derivative [1]-[3], [8]-[12]. More precisely, the symmetric logarithmic derivative of the state $\rho$ with respect to an observable $H_{\alpha}$ is a self-adjoint operator $L_{\alpha}$ determined by

$$
D_{H_{\alpha}} \rho=\frac{1}{2}\left(L_{\alpha} \rho+\rho L_{\alpha}\right), \quad \alpha=1, \ldots, n .
$$

Now following [8], [9] and mimicking the classical expression (7), we can define the quantum Fisher information matrix $\mathbf{F}:=\left(F_{\alpha \beta}\right)_{1 \leqslant \alpha, \beta \leqslant n}$ (based on the symmetric logarithmic derivative) as

$$
F_{\alpha \beta}:=\operatorname{tr} \rho L_{\alpha} L_{\beta}, \quad \alpha, \beta=1, \ldots, n .
$$

This matrix is apparently positive. It plays a central role in the theory of quantum estimation for shift parameters.

3. A matrix inequality and uncertainty relations. Our main result is the following inequality relating the covariance matrix $\mathbf{C}$ defined by (1) and the quantum Fisher information matrix defined by (8).

Theorem. We have $\mathbf{C} \geqslant \frac{1}{4} \mathbf{F}$. In particular,

$$
V\left(\rho, H_{1}\right) V\left(\rho, H_{2}\right)-\left|\operatorname{cov}_{\rho}\left(H_{1}, H_{2}\right)\right|^{2} \geqslant \frac{1}{16}\left(F_{11} F_{22}-\left|F_{12}\right|^{2}\right) .
$$

$\mathrm{P}$ r o of. Suppose that the dimension of the Hilbert space is $d$, and the spectral decomposition of $\rho$ is $\rho=\sum_{j=1}^{d} \lambda_{j}\left|\psi_{j}\right\rangle\left\langle\psi_{j}\right|$. Here $\left\{\left|\psi_{j}\right\rangle\right\}$ is the set of eigenvectors of $\dot{\rho}$ with corresponding eigenvalues $\left\{\lambda_{j}\right\}$ (counting multiplicity). We may further assume that $\left\{\left|\psi_{j}\right\rangle\right\}$ constitutes an orthonormal base for the Hilbert space, and thus we have the resolution of identity

$$
\sum_{j=1}^{d}\left|\psi_{j}\right\rangle\left\langle\psi_{j}\right|=1
$$

For $\alpha=1, \ldots, n$, put $\left\langle H_{\alpha}\right\rangle:=\operatorname{tr} \rho H_{\alpha}$ and let $h_{j k}^{(\alpha)}:=\left\langle\psi_{j}\left|H_{\alpha}-\left\langle H_{\alpha}\right\rangle \mathbf{1}\right| \psi_{k}\right\rangle, j, k=$ $1, \ldots, d$, be the matrix elements of the operator $H_{\alpha}-\left\langle H_{\alpha}\right\rangle 1$ in the representation with respect to the orthonormal base $\left\{\left|\psi_{j}\right\rangle\right\}$. Clearly, $\overline{h_{j k}^{(\alpha)}}=h_{k j}^{(\alpha)}$.

By the defining equation (1),

$$
C_{\alpha \beta}=\operatorname{tr} \rho\left(H_{\alpha}-\left\langle H_{\alpha}\right\rangle 1\right)\left(H_{\beta}-\left\langle H_{\beta}\right\rangle 1\right)=\sum_{j=1}^{d}\left\langle\psi_{j}\left|\rho\left(H_{\alpha}-\left\langle H_{\alpha}\right\rangle 1\right)\left(H_{\beta}-\left\langle H_{\beta}\right\rangle 1\right)\right| \psi_{j}\right\rangle
$$

$$
=\sum_{j=1}^{d} \lambda_{j}\left\langle\psi_{j}\left|\left(H_{\alpha}-\left\langle H_{\alpha}\right\rangle \mathbf{1}\right)\left(H_{\beta}-\left\langle H_{\beta}\right\rangle \mathbf{1}\right)\right| \psi_{j}\right\rangle
$$

by (10) $=\sum_{j=1}^{d} \lambda_{j}\left\langle\psi_{j}\left|\left(H_{\alpha}-\left\langle H_{\alpha}\right\rangle 1\right) \sum_{k=1}^{d}\right| \psi_{k}\right\rangle\left\langle\psi_{k}\left|\left(H_{\beta}-\left\langle H_{\beta}\right\rangle \mathbf{1}\right)\right| \psi_{j}\right\rangle$

$$
=\sum_{j, k=1}^{d} \lambda_{j} h_{j k}^{(\alpha)} h_{k j}^{(\beta)}=\sum_{j, k=1}^{d} \lambda_{j} h_{j k}^{(\alpha)} \overline{h_{j k}^{(\beta)}} .
$$


On the other hand, from $\left[\rho, H_{\alpha}\right]=\left[\rho, H_{\alpha}-\left\langle H_{\alpha}\right\rangle 1\right]$ and $i\left[\rho, H_{\alpha}\right]=\frac{1}{2}\left(L_{\alpha} \rho+\rho L_{\alpha}\right)$, $\alpha=1, \ldots, n$, we have

$$
\left\langle\psi_{j}\left|i \rho\left(H_{\alpha}-\left\langle H_{\alpha}\right\rangle 1\right)-\left(H_{\alpha}-\left\langle H_{\alpha}\right\rangle 1\right) \rho\right| \psi_{k}\right\rangle=\frac{1}{2}\left\langle\psi_{j}\left|L_{\alpha} \rho+\rho L_{\alpha}\right| \psi_{k}\right\rangle, \quad j, k=1, \ldots, d .
$$

That is, $i\left(\lambda_{j}-\lambda_{k}\right)\left\langle\psi_{j} \mid H_{\alpha}-\left\langle H_{\alpha}\right\rangle 1_{k}\right\rangle=\frac{1}{2}\left(\lambda_{j}+\lambda_{k}\right)\left\langle\psi_{j}\left|L_{\alpha}\right| \psi_{k}\right\rangle$ which implies

$$
\left\langle\psi_{j}\left|L_{\alpha}\right| \psi_{k}\right\rangle=\frac{2 i\left(\lambda_{j}-\lambda_{k}\right)}{\lambda_{j}+\lambda_{k}} h_{j k}^{(\alpha)}
$$

Now by the defining equation (8),

$$
F_{\alpha \beta}=\operatorname{tr} \rho L_{\alpha} L_{\beta}=\sum_{j=1}^{d}\left\langle\psi_{j}\left|\rho L_{\alpha} L_{\beta}\right| \psi_{j}\right\rangle=\sum_{j=1}^{d} \lambda_{j}\left\langle\psi_{j}\left|L_{\alpha} L_{\beta}\right| \psi_{j}\right\rangle
$$

by (10) $\quad=\sum_{j=1}^{d} \lambda_{j}\left\langle\psi_{j}\left|L_{\alpha} \sum_{k=1}^{d}\right| \psi_{k}\right\rangle\left\langle\psi_{k}\left|L_{\beta}\right| \psi_{j}\right\rangle=\sum_{j, k=1}^{d} \lambda_{j}\left\langle\psi_{j}\left|L_{\alpha}\right| \psi_{k}\right\rangle\left\langle\psi_{k}\left|L_{\beta}\right| \psi_{j}\right\rangle$

by (11) $=\sum_{j, k=1}^{d} \lambda_{j} \frac{4}{\left(\lambda_{j}+\lambda_{k}\right)^{2}}\left(\lambda_{j}-\lambda_{k}\right)^{2} h_{j k}^{(\alpha)} \overline{h_{j k}^{(\beta)}}$.

Therefore,

$$
\begin{aligned}
C_{\alpha \beta}-\frac{1}{4} F_{\alpha \beta} & =\sum_{j, k=1}^{d} \lambda_{j} h_{j k}^{(\alpha)} \overline{h_{j k}^{(\beta)}}-\frac{1}{4} \sum_{j, k=1}^{d} \lambda_{j} \frac{4}{\left(\lambda_{j}+\lambda_{k}\right)^{2}}\left(\lambda_{j}-\lambda_{k}\right)^{2} h_{j k}^{(\alpha)} \overline{h_{j k}^{(\beta)}} \\
& =\sum_{j, k=1}^{d} \frac{4 \lambda_{j}^{2} \lambda_{k}}{\left(\lambda_{j}+\lambda_{k}\right)^{2}} h_{j k}^{(\alpha)} \overline{h_{j k}^{(\beta)}} .
\end{aligned}
$$

Consequently,

$$
\mathbf{C}-\frac{1}{4} \mathbf{F}=\sum_{j, k=1}^{d} \frac{4 \lambda_{j}^{2} \lambda_{k}}{\left(\lambda_{j}+\lambda_{k}\right)^{2}}\left(\begin{array}{c}
h_{j k}^{(1)} \\
\vdots \\
h_{j k}^{(n)}
\end{array}\right)\left(\overline{h_{j k}^{(1)}}, \ldots, \overline{h_{j k}^{(n)}}\right) .
$$

Since each summand matrix is a rank one projection (modulo a positive constant) and thus positive, it follows that $\mathbf{C}-\frac{1}{4} \mathbf{F}$ is positive and accordingly $\mathbf{C} \geqslant \frac{1}{4} \mathbf{F}$.

Now because both $\mathbf{C}$ and $\mathbf{F}$ are positive, by taking determinant of both sides of $\mathbf{C} \geqslant \frac{1}{4} \mathbf{F}$, we have

$$
\operatorname{det} \mathbf{C} \geqslant \operatorname{det}\left(\frac{1}{4} \mathbf{F}\right)
$$

In particular, when $n=2$, we have

$$
\operatorname{det} \mathbf{C}=V\left(\rho, H_{1}\right) V\left(\rho, H_{2}\right)-\left|\operatorname{cov}_{\rho}\left(H_{1}, H_{2}\right)\right|^{2}, \quad \operatorname{det}\left(\frac{1}{4} \mathbf{F}\right)=\frac{1}{16}\left(F_{11} F_{22}-\left|F_{12}\right|^{2}\right)
$$

Inequality (9) follows immediately.

Finally, we remark that $\operatorname{det}\left(\frac{1}{4} \mathbf{F}\right) \geqslant 0$ and, in general, the strict inequality holds. Thus the uncertainty relation (9) is stronger than the Schrödinger uncertainty relation (6).

4. Conclusion. We have established a matrix inequality connecting the covariance matrix and the quantum Fisher information matrix defined via the symmetric logarithmic derivative. This inequality has two natural interpretations dual to each other: It can be regarded either as that the information matrix places a lower bound to the covariance matrix, or as that the covariance matrix sets an upper bound to the information matrix. Both are in the spirit of the uncertainty principle and in fact can be regarded as particular quantifications of it. As an interesting consequence, we readily obtain an uncertainty relation which improves the Schrödinger uncertainty relation. 
Acknowledgments. The author is very grateful to the referee for pointing out the rigorous interpretation of the quantum covariance matrix and the uncertainty relation.

\section{СПИСОК ЛИТЕРАТУРЫ}

1. Белавжин В. П. Об обобшенных соотношениях неопределенности Гейзенберга и эффективных измерениях в квантовых системах. - Теорет. матем. физ., 1976, т. 26, № 3, c. 316-329.

2. Barndorff-Nielsen O.E., Gill R.D. Fisher information in quantum statistics. J. Phys. A, 2000, v. 33, № 24, pp. 4481-4490.

3. Barndorff-Nielsen O.E., Gill R.D., Jupp P.E. On quantum statistical inference. J. Roy. Statist. Soc. Ser. B, 2003, v. 65, № 4, p. 775-816.

4. Connes A. Noncommutative Geometry. San Diego: Academic Press, 1994, 661 p.

5. Крамер Г. Математические методы статистики. М.: Мир, 1975, 648 с.

6. Дирак П. Принципы квантовой механики. М.: Физматгиз, 1960, $434 \mathrm{c.}$

7. Fisher R. A. Theory of statistical estimation. - Proc. Cambridge Philos. Soc., 1925, v. 22 , p. $700-725$.

8. Helstrom C. W. Quantum Detection and Estimation Theory. New York: Academic Press, 1976.

9. Холево A.C. Вероятностные и статистические аспекты квантовой теории. М.-Ижевск: Ин-т компьют. исслед., 2003, 404 с.

10. Холево A.C. Асимптотическое оценивание параметра сдвига квантового состояния. - Теория вероятн. и ее примен., 2004 , т. 49 , в. 2 , с. $335-350$.

11. Luo $S$. Wigner-Yanase skew information vs. quantum Fisher information. - Proc. Amer. Math. Soc., 2004, v. 132, № 3, p. 885-890.

12. Petz D. Monotone metrics on matrix spaces. - Linear Algebra Appl., 1996, v. 244, p. 81-96.

13. Sakurai J. J. Modern Quantum Mechanics. Reading, MA: Addison-Wesley, 1994.

14. Schrödinger E. About Heisenberg uncertainty relation. - Proc. Prussian Acad. Sci., Phys.-Math. Sect., 1930, v. 19, p. 296-303; pyc. пер.: К принципу неопределенности Гейзенберга. - Э. Шредингер. Избранные труды по квантовой механике. М.: Наука, 1976, с. 210-217.

15. Фон Нейман Дж. Математические основы квантовой механики. М.: Наука, 1964, $367 \mathrm{c}$.

Поступила в редакцию

14.XI.2005

Исправленный вариант

6.VI.2007

(c) 2008 г.

MATTNER L.*

\section{LOWER BOUNDS FOR TAILS OF SUMS OF INDEPENDENT SYMMETRIC RANDOM VARIABLES ${ }^{1)}$}

Обобщая подход Клейтмана [9] и Кантера [8] к неравенствам для многомерных функций концентрации, мы получаем для вероятностей уклонений сумм независимых симметричных случайных величин оценку снизу, зависящую только от вероятностей уклонений слагаемых. Эта оценка оптимальна с точностью до эффектов дискретизации, улучшает результат Нагаева [11] и дополняет теоремы сравнения Бирнбаума [3] и Прусса [12]. Терема Бирнбаума для унимодальных случайных величин распространяется на решетчатый случай.

* Universität zu Lübeck, Institut für Mathematik, Wallstr. 40, D-23560 Lübeck, Germany; e-mail: mattner@math.uni-luebeck.de 